\title{
Further Characterization of Cytochrome P-460 in Nitrosomonas europaea
}

\author{
By DAVID J. MILLER, 1.2 PAUL M. WOOD' AND D. J. D. NICHOLAS 2* \\ 'Department of Biochemistry', Medical School, Unicersity of Bristol, University Walk. \\ Bristol BS8 ITD, UK \\ 2Department of Agricultural Biochemistry, Waite Agricultural Research Institute, \\ University of Adelaide. Glen Osmond. South Australia 5064
}

(Received 16 May 1984 ; revised 9 July 1984)

\begin{abstract}
Reduction of cells or extracts of Nitrosomonas europaea with dithionite leads to a peak in absorption spectra at $460 \mathrm{~nm}$. Most of this chromophore is bound to hydroxylamine oxidase, but a small fraction exists as a separate protein, namely cytochrome P-460. An improved purification of both these proteins is described. The mobility of cytochrome $\mathbf{P} \mathbf{- 4 6 0}$ on gel electrophoresis in the presence of SDS corresponded to a molecular weight of 18000 , whereas a molecular weight of 52000 was determined by gel filtration. The band obtained by electrophoresis gave a yellowgreen fluorescence in UV light. Electrophoresis in the presence of $8 \mathrm{M}$-urea, or after acid-treatment, resulted in red fluorescence from cytochrome P.460.

We previously reported that extracts from $N$. europaea gave eight fluorescence bands in SDSpolyacrylamide gels; cytochrome $\mathrm{P}-\mathbf{4 6 0}$ was found to correspond to the previously unassigned band VI. Electrophoresis of purified hydroxylamine oxidase did not give a band in common with cytochrome P-460. Highly purified cytochrome P-460 gave no detectable flavin-type fluorescence. There was no antigenic similarity between hydroxylamine oxidase and cytochrome P-460.
\end{abstract}

\section{INTRODUCTION}

The absorption spectra of cells or extracts of ammonia-oxidizing bacteria such as Nitrosomonas europaea have the distinctive feature of a peak at $460 \mathrm{~nm}$ in the presence of dithionite. The chromophore responsible for this absorption has been named P-460 by analogy with cytochrome $P-450$, although it should be emphasized that the long-wavelength absorption peak does not require the presence of a ligand such as CO (Rees \& Nason, 1965; Hooper et al., 1978). The major source of $P-460$ is hydroxylamine oxidase (EC 1.7.3.4), a complex protein with multiple $c$-type haems (Lipscomb \& Hooper, 1982). The P-460 redox centre has been implicated in binding both hydroxylamine and oxygen, since its selective destruction by hydrogen peroxide results in loss of the ability of hydroxylamine to reduce the protein and loss of autooxidizability of the reduced $c$-type haems (Hooper \& Terry, 1977; Hooper \& Balny, 1982). Hydroxylamine oxidase has a molecular weight of $180000-200000$ and a spectrum dominated by absorption by the $c$-type haems (Hooper et al., 1978; Yamanaka et al., 1979). By contrast, Erickson \& Hooper (1972) described the isolation of a less abundant protein with absorption changes similar to P-460 in hydroxylamine oxidase, but lacking any other chromophore. Its mobility on gel filtration corresponded to a molecular weight of 52000 . We shall refer to this protein as cytochrome $P-460$.

We have recently used the fluorescent gel technique (Wood, 1981) to characterize the $c$-type cytochromes in $N$. europaea (Miller \& Wood, 1982, 1983). Unfractionated soluble extracts gave rise to eight bands of fluorescence, for which the following assignments were made: I and II, hydroxylamine oxidase: III, cytochromes $c_{C O}-550$ and $c-553$; IV, cytochrome $c-554$; VII, cytochrome $c_{\mathrm{CO}}-552$; VIII, cytochrome $c-552$. This paper describes an improved purification procedure for cytochrome P-460, so that it can now be shown to correspond with band VI on the fluorescent gels, and also considers its relationship to hydroxylamine oxidase. 


\section{METHODS}

Details of the bacterium, cell growth and harvesting, spectroscopy, protein assay and SDS-PAGE were as previously described (Miller \& Wood, 1982). Electrophoresis in SDS plus $8 \mathrm{M}$-urea was conducted as described by Swank \& Munkres (1971) except that samples were denatured prior to electrophoresis by boiling for $2 \mathrm{~min}$ in $8 \mathrm{M}$ urea plus $2 \%(w / v)$ SDS and $15 \%(v / v) 2$-mercaptoethanol.

Determination of flavin by fluorescence spectroscopy was conducted as described by Siegel (1978) using a Baird SF-1 fluorescence spectrophotometer (Baird-Atomic, Cambridge, Mass., USA).

Hydroxylamine oxidase. This was purified as previously described (Miller \& Wood, 1982) but with the addition of a further ion-exchange chromatography step. The eluate from the first DEAE-cellulose column was dialysed overnight at $4^{\circ} \mathrm{C}$ against $4110 \mathrm{~mm}$-Tris/HCl buffer, $\mathrm{pH} \mathrm{8.0,} \mathrm{and} \mathrm{loaded} \mathrm{onto} \mathrm{a} \mathrm{short} \mathrm{column}(5 \times 1.2 \mathrm{~cm})$ of DEAE-cellulose in the same buffer. The column was washed with $40 \mathrm{ml}$ buffer and then eluted with a $400 \mathrm{ml}$ linear gradient of buffer plus $0-200 \mathrm{~mm}-\mathrm{NaCl}$. The hydroxylamine oxidase obtained consistently gave $A_{410}(0 x i d i z e d): A_{280}=4 \cdot 3$, better than the value of 3.0 cited for the purified protein by previous authors (Hooper et al., 1978; Yamanaka et al., 1979).

Antibodies to purified hydroxylamine oxidase were raised in rabbits. Samples of protein $(0.5 \mathrm{mg})$ were homogenized with equal volumes of Freund's complete adjuvant and injected subcutaneously at multiple sites into $2 \mathrm{~kg}$ male New Zealand White rabbits on three occasions at 7-d intervals. A fourth injection was given after a further $14 \mathrm{~d}$, and at 7 and $14 \mathrm{~d}$ after the final injection the rabbits were bled from the terminal ear vein. The blood was allowed to clot for $6 \mathrm{~h}$ at room temperature, then stored at $4{ }^{\circ} \mathrm{C}$ overnight. Clots were removed by centrifugation $(18000 \mathrm{~g}, 20 \mathrm{~min})$, and the serum partially fractionated as described by Chua \& Blomberg (1979) except that IgG was not separated from other immunoglobulins.

Cytochrome P-460. For purification of cytochrome $\mathrm{P}-460$ the pellet from the $75-90 \%$ saturation $\left(\mathrm{NH}_{4}\right)_{2} \mathrm{SO}_{4}$ fraction of Miller \& Wood (1982) was used. The pellet was redissolved in a small volume of $10 \mathrm{mM}-\mathrm{Tris} / \mathrm{HCl}$ buffer, pH 8.0, and chromatographed on a column of Sephacryl S-200(80 $\times 5 \mathrm{~cm})$ equilibrated with this buffer. Elution of P-460 was monitored at $A_{43}$ s. It was eluted as a green peak, with mobility corresponding to a molecular weight of 52000. Peak fractions were loaded directly onto a short column $(7 \times 1.2 \mathrm{~cm})$ of DEAE-cellulose (Whatman DE52) equilibrated with the same buffer. A $400 \mathrm{ml}$ linear gradient of buffer plus $0-500 \mathrm{~mm}-\mathrm{NaCl}$ was applied, and cytochrome P-460 was eluted at about $75 \mathrm{~mm}-\mathrm{NaCl}$. Cytochrome $\mathrm{P}-460$ peak fractions from ion-exchange chromatography were pooled, dialysed and concentrated by freeze-drying. This material was used for spectroscopy. Homogeneous cytochrome $P-460$ was prepared from the ion-exchange eluate by preparative SDSPAGE. Approximately $600 \mu \mathrm{g}$ protein was denatured as previously described (Wood, 1981) and then applied to a $15 \times 12 \times 0.1 \mathrm{~cm} \mathrm{slab} \mathrm{gel} \mathrm{(15 \% ,} \mathrm{w/v,} \mathrm{resolving} \mathrm{gel;} 5 \%, \mathrm{w} / \mathrm{v}$, stacking gel) and subjected to electrophoresis for $4 \mathrm{~h}$ at $30 \mathrm{~mA}$. A gel section was then rapidly stained for protein, the band corresponding in mobility with cytochrome $\mathrm{P}-460$ excised from the remainder of the gel, and the gel slice finely diced into $2 \mathrm{ml} 10 \mathrm{mM}-\mathrm{Tris} / \mathrm{HCl}, \mathrm{pH} \mathrm{8.0}$. The protein was allowed to diffuse out of the gel slices overnight at $4^{\circ} \mathrm{C}$ with stirring (at this stage recovery was at least $60 \%$. Recovered protein was concentrated by dialysis against saturated sucrose solution. Antibodies to cytochrome $P-460$ which had been purified in this way were raised in rabbits as described above, except that approximately $100 \mu \mathrm{g}$ protein was used per injection. Unfractionated serum was used as the source of antibodies against cytochrome P-460 in immunodiffusion experiments.

\section{RESULTS}

\section{Electrophoresis in the presence of SDS}

A sample of cytochrome P-460 (ion-exchange eluate) was subjected to SDS-PAGE. Illumination of the gel with near-UV light showed a single band of fluorescence, yellowish-green in colour; see Fig. 1(a). The band corresponded in mobility and fluorescence colour to band VI of Miller \& Wood (1982) for which an apparent molecular weight of 18000 was assigned. Protein staining with Coomassie blue revealed minor contaminants in the $40000-45000$ molecular weight range (Fig. $1 \mathrm{~b}$ ), but the major protein band ( $>90 \%$ of the stain) corresponded to the band of fluorescence. After electrophoretic purification, re-electrophoresis of cytochrome P-460 gave one major band staining with Coomassie blue; trace contaminants of low molecular weight were occasionally observed, but these were assumed to be proteolytic fragments of cytochrome $P-460$. Insufficient homogeneous cytochrome $P-460$ was available for determination of its fluorescence characteristics after SDS-PAGE.

We previously reported that the fluorescent bands produced after electrophoresis of hydroxylamine oxidase included a yellow-green band VI (Miller \& Wood, 1982), but this band was absent from the highly purified hydroxylamine oxidase prepared as described above. The 


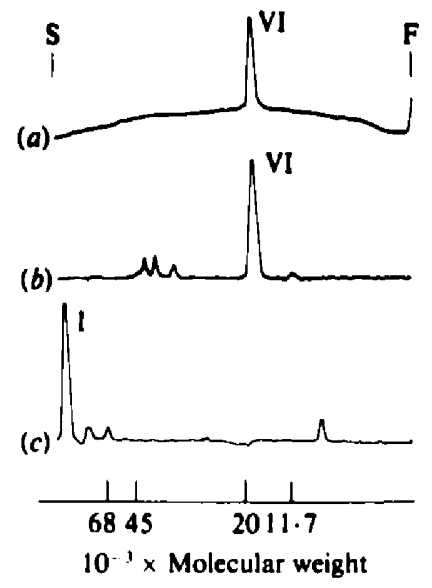

Fig. 1. Electrophoresis of cytochrome P-460 and hydroxylamine oxidase. Sample treatment and electrophoresis were conducted as previously described (Miller \& Wood, 1982). (a) Densitometer scan of a photographic negative exposed to fluorescence from SDS-polyacrylamide rod gel loaded with $200 \mu \mathrm{g}$ cytochrome P-460. (b) Densitometer scan of the same gel after staining for protein with Coomassic blue. (c) As (b), but with a loading of approx. $100 \mu \mathrm{g}$ hydroxylamine oxidase in place of cytochrome P-460. The positions of four molecular weight markers are shown: bovine albumin (68000); ovalbumin (45000); soybean trypsin inhibitor (20000); cytochrome $c$ from horse heart (11 700). S, start of resolving gel; F, dye front. Band assignment (I and VI) was as in our earlier paper (Miller \& Wood, 1982).

same material also gave no band on protein staining that matched any from cytochrome P-460; see Fig. $1(c)$.

Electrophoresis of cytochrome P-460 (ion-exchange eluants) in SDS plus 8 M-urea gave a similar mobility for the fluorescent band but no protein band in common between cytochrome $P-460$ and hydroxylamine oxidase. However, the fluorescence from cytochrome P-460 was not yellow-green but red. Conversion of cytochrome P-460 to a form giving red fluorescence was also achieved by the use of strongly acid denaturation conditions (Ward et al., 1983) or by exposure to acid (pH 4 for $24 \mathrm{~h}$ at $4^{\circ} \mathrm{C}$ ), followed by standard SDS-PAGE. Breakage of cells by rapid freezing in liquid $\mathrm{N}_{2}$ followed by thawing and protein purification as described above also yielded a form of cytochrome P-460 giving red fluorescence after SDS-PAGE. The significance of these results is discussed below.

\section{Spectroscopy of P-460}

Fig. 2 (a) shows the absorption spectrum of purified cytochrome $P-460$. The rato $A_{435}: A_{280}$ was 0.6 , as against 0.1 in the similar spectra of Erickson \& Hooper (1972), indicating that our material was of considerably higher purity. A difference spectrum of reduced-plus- $\mathrm{CO}$ minus reduced (Fig. 2c) showed a trough at $460 \mathrm{~nm}$, as reported by Erickson \& Hooper (1972). A similar trough was obtained with hydroxylamine oxidase; see Fig. $2(b)$. In an earlier paper (Miller \& Wood, 1982) the green fluorescence from band VI on electrophoretograms of $N$. europaea extracts (cytochrome P-460) was suggested to be due to flavin. However, both purified cytochrome P-460 (ion-exchange eluate) and hydroxylamine oxidase were shown to be free of flavin-type fluorescence (Fig. $2 d-f$ ). Fluorescence spectroscopy over a wide range of protein concentrations indicated that both these purified proteins contained less than $0.005 \mathrm{~mol}^{\text {flavin mol}}{ }^{-1}$, hence unless extremely strong internal fluorescence quenching occurred they can be considered to be devoid of flavin.

\section{Antigenic cross reactivity}

In Ouchterlony double diffusion experiments antibodies raised to highly purified hydroxylamine oxidase gave a single strong precipitation line when run against either hydroxylamine oxidase or soluble preparations of $N$. europaea, but no precipitation reaction 

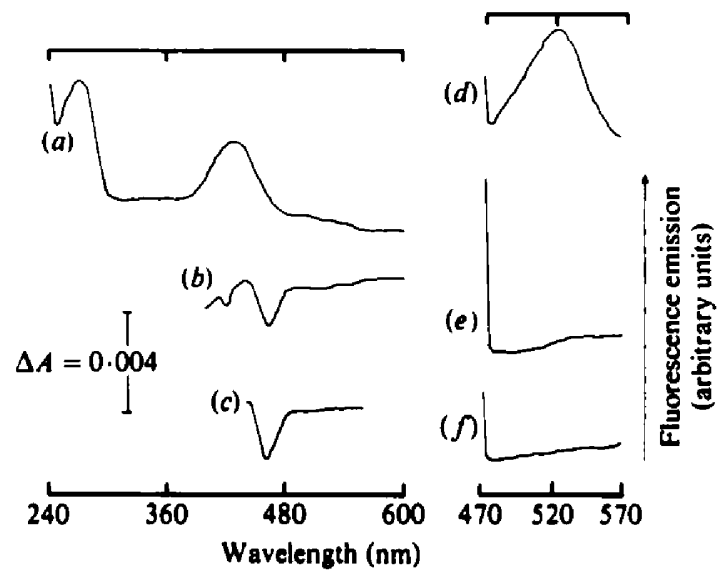

Fig. 2. Spectroscopy of P-460. Spectroscopy was conducted as previously described (Miller \& Wood, 1982) using Pye-Unicam SP8-200 or Perkin-EImer $\lambda$-5 split-beam spectrophotometers. Fluorescence spectroscopy was conducted as described in Methods. (a) Absolute spectrum of purified cytochrome P-460. The sample cuvette contained oxidized cytochrome P-460 in $20 \mathrm{mM}-\mathrm{MOPS} / \mathrm{KOH}$ buffer, pH 7.0, plus $100 \mathrm{mu}-\mathrm{KCl}$ (protein concentration $10 \mu \mathrm{g} \mathrm{ml} \mathrm{m}^{-1}$ ). The reference cuvette contained buffer alone. (b) CO-difference spectrum for reduced hydroxylamine oxidase. Both cuvettes contained purified hydroxylamine oxidase, in buffer as for $(a)$. A few grains of solid dithionite were added to both cuvettes and $\mathrm{CO}$ was bubbled into the sample cuvette (protein concentration $30 \mu \mathrm{g} \mathrm{ml}-1$ ). (c) CO-difference spectrum for reduced cytochrome $P-460$. Conditions were as in (b), except for cytochrome $P-460$ in place of hydroxylamine oxidase (protein concentration $16 \mu \mathrm{g} \mathrm{ml} \mathrm{l}^{-1}$ ). (d) Fluorescence emission spectrum (excitation at $450 \mathrm{~nm}$ ) for $40 \mathrm{nM}-\mathrm{FMN}$ in $50 \mathrm{~mm}-\mathrm{HEPES} / \mathrm{NaOH}$ buffer, $\mathrm{pH} 7.7$. (e) Fluorescence emission spectrum for cytochrome $\mathrm{P}-460\left(50 \mu \mathrm{g} \mathrm{ml} \mathrm{m}^{-1}\right)$ under conditions as in (d). ( $f$ ) Fluorescence emission spectrum for hydroxylamine oxidase $(50 \mu \mathrm{g} \mathrm{ml}-1)$ under conditions as in $(d)$ and $(e)$.
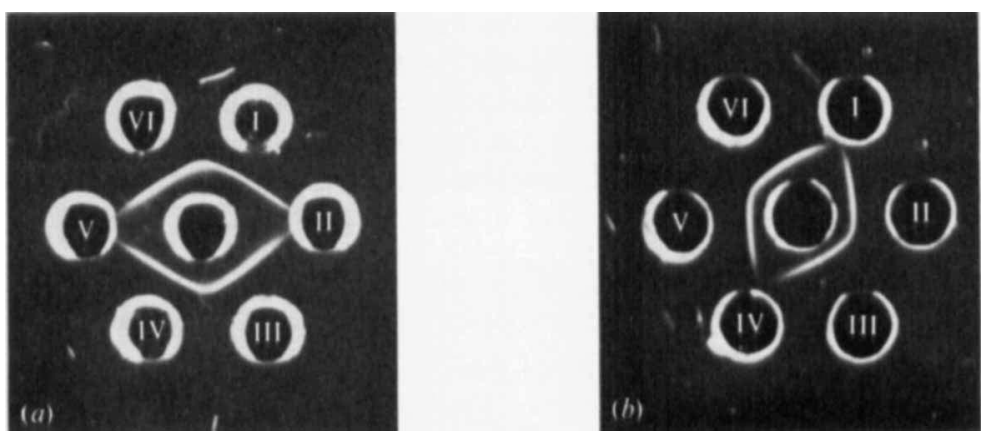

Fig. 3. Immunodiffusion of antibodies to hydroxylamine oxidase and cytochrome $\mathbf{P}-\mathbf{4 6 0}$. The figure shows results of double diffusion experiments in which the centre well contained either $(a) 5 \mu l$ antihydroxylamine oxidase (immunoglobulin fraction), or (b) $20 \mu \mathrm{l}$ anti-P-460 (whole serum). In each the outer wells contained: I and IV, $5 \mu \mathrm{g}$ hydroxylamine oxidase; II and V, $5 \mu \mathrm{g}$ cytochrome P-460; III and VI, $40 \mu \mathrm{g}$ whole soluble $N$. europaea extract. Immunodiffusion was conducted at $20{ }^{\circ} \mathrm{C}$ in $1 \%(w / v)$ agarose containing $50 \mathrm{~mm}-\mathrm{Tr} / \mathrm{HCl}, \mathrm{pH} 8.0$ and $0.1 \mathrm{M}-\mathrm{NaCl}$. The immunodiffusion plates were photographed without staining, using Kodak AHU 5460 microfilm.

against purified cytochrome P-460 (Fig. 3a). This result occurred with two batches of antibody raised to two distinct hydroxylamine oxidase preparations. Even after $10 \mathrm{~d}$ no precipitation reaction between antibodies to hydroxylamine oxidase and purified cytochrome P-460 appeared.

The antibody titre of antiserum raised to cytochrome $P-460$ was low, but there was clearly no precipitation reaction with hydroxylamine oxidase (Fig. $3 b$ ). Occasional weak secondary precipitation lines were observed upon immunodiffusion of antiserum to P-460 against $P-460$ preparations or soluble fractions. 


\section{DISCUSSION}

The distinctive feature of $460 \mathrm{~nm}$ in spectra for whole cells or soluble extracts of $N$. europaea is predominantly associated with hydroxylamine oxidase, but we estimate about $5 \%$ to be attributable to cytochrome $P-460$. This cytochrome is responsible for the yellow-green fluorescent band VI on SDS-PAGE which remained unassigned in our earlier studies (Miller \& Wood, 1982, 1983); thus only one band on such gels now remains to be identified. On gel filtration, cytochrome P-460 ran at a molecular weight of 52000 (see Methods); comparison with the apparent molecular weight of 18000 on SDS-PAGE indicates that in the natural state it is a trimer. Erickson \& Hooper (1972) also gave a molecular weight value of 52000 for the native cytochrome $P-460$ preparations, but subsequently corrected this value to 36000 to account for a high (30\%) RNA contaminant. As judged by $280: 260 \mathrm{~nm}$ absorption ratio (Cherry, 1973), our preparations contained less than $2 \%$ RNA/DNA, so that a correction was not necessary. Electrophoresis of highly purified hydroxylamine oxidase gave no band in common with cytochrome $\mathrm{P}-460$, nor was there any antigenic identity. The unusual spectral features at $460 \mathrm{~nm}$ are therefore due to two distinct proteins. Hooper and his co-workers (Hooper et al., 1978; Terry \& Hooper, 1981) likewise found that electrophoresis of highly purified hydroxylamine oxidase, under a variety of conditions, did not produce subunits in the molecular weight range of 17-18000. However, Hooper (1984), citing unpublished results, indicated that antibodies to hydroxylamine oxidase reacted with $\mathrm{P}-\mathbf{4 6 0}$ preparations. This discrepancy with our results probably arises because our preparations are of higher purity (see Methods). Clarification of the relationship between the two proteins may require selective proteolysis and protein sequencing; the prosthetic group may be inserted by a specific enzyme, as is the case for cytochrome $c$, in which case both hydroxylamine oxidase and cytochrome $P-460$ would have recognition sites.

The chemical nature of the $\mathbf{P}-\mathbf{4 6 0}$ chromophore remains unclear. It is thought to be haem because it has a Soret-type absorption peak when reduced and binds typical haem ligands such as CO (Lipscomb \& Hooper, 1982). The Mössbauer spectra obtained for hydroxylamine oxidase support this conclusion (Lipscomb et al., 1982). The haem is not extractable with acid acetone (Yamanaka et al., 1979; Erickson \& Hooper, 1972), and evidently remained attached on boiling with SDS. These observations point to covalent linkage as in c-type cytochromes. Lactoperoxidase and myeloperoxidase are examples of haem proteins with long-wavelength Soret bands and covalent bonds between haem and protein; in both cases the haem structure is unusual (Odajima \& Yamazaki, 1972; Wu \& Schultz, 1975). Chemical modification of the covalently bound haem in cytochrome P-460 may be responsible for the observed change in fluorescence colour from yellow-green to red with modified sample treatment conditions described above. More precise chemical characterization awaits isolation of larger quantities of cytochrome P-460, which will permit isolation of the chromophore and determination of the linkage to the peptide chain.

We thank the Science and Engineering Research Council, UK, and the Australian Research Grants Scheme for research grants. The technical assistance of Dr T. Hopkirk and Mr T. Howell is gratefully acknowledged.

\section{REFERENCES}

Cherrey, J. (1973). Molecular Biology of Plants : a Textmanual, p. 172. New York: Columbia University Press.

Chua, N.-H. \& Blomberg, F. (1979). Immunochemical studies of thylakoid membrane polypeptides from spinach and Chlamydomonas reinhardii. Journal of Biological Chemistry 254, 215-223.

Ericxson, R. H. \& Hooper, A. B. (1972). Preliminary characterization of a variant CO-binding heme protein from Nitrosomonas. Biochimica et biophysica acra 275, 231-244.

HoOper, A. B. (1984). Ammonia oxidation and energy transduction in the nitrifying bacteria. In Microbial
Chemoautotrophy. Edited by W. R. Strohl \& O. H. Tuovinen. Columbus, USA: Ohio State University Press.

HOOPER, A. B. \& BALNY, C. (1982). Reaction of oxygen with hydroxylamine oxidoreductase of Nitrosomonas. Fast kinetics. FEBS Letters 144, 299-303.

HOOPER, A. B. \& TeRRY, K. R. (1977) Hydroxylamine oxidoreductase from Nitrosomonas: inactivation by hydrogen peroxide. Biochemistry 16, 455-459.

HoOper, A. B., MaXwell, P. C. \& Terry, K. R. (1978). Hydroxylamine oxidoreductase from Nitrosomonas: absorption spectra and content of heme and metal. Biochemistry 17, 2984-2989. 
Lipscomb, J. D. \& HoOPER, A. B. (1982). Resolution of multiple heme centers of hydroxylamine oxidoreductase from Nitrosomonas. 1. Electron paramagnetic resonance spectroscopy. Biochemistry 21, 39653972.

Lipsconb, J. D., Andersson, K. K., MÜnCK, E., KENT, T. A. \& HOOPER, A. B. (1982). Resolution of multiple heme centers of hydroxylamine oxidoreductase from Nitrosomonas. 2. Mőssbauer Spectroscopy. Biochemistry 21, 3973-3976.

Miller, D. J. \& Wood, P. M. (1982). Characterization of the c-type cytochromes of Nitrosomonas europaea with the aid of fluorescent gels. Biochemical Journal 207, 511-517.

Millex, D. J. \& WoOD, P. M. (1983). CO-binding ctype cytochromes and a high potential cytochrome $c$ in Nitrosomonas europaea. Biochemical Journal 211, 503-506.

ODAIMA, T. \& YamzakI, I. (1972). Myeloperoxidase of the leukocyte of normal blood. IV. Some physicochemical properties. Biochimica et biophysica acta 284, 360-367.

ReEs, M. K. \& NASON, A. (1965). A P-450-like cytochrome and a soluble terminal oxidase identified as cytochrome of from Nitrosomonas europaea. Biochemical and Biophysical Research Communications 21, 248-256.
SirorL, L. M. (1978). Quantitative determination of noncovalently bound flavins: types and methods of analysis. Methods in Enzymology 53, 419-429.

SWANK, R. T. MUNKREs, K. D. (1971). Molecular weight analysis of oligopeptides by electrophoresis in polyacrylamide gel with sodium dodecyl sulfate. Analytical Biochemistry 39, 462-477.

TERRY, K. R. HOOPER, A. B. (1981). Hydroxylamine oxidoreductase: a 20-heme, 200000 molecular weight cytochrome $c$ with unusual denaturation properties which forms a 63000 molecular weight monomer after heme removal. Biochemistry 20 , 7026-7032.

W ARD, J. A., HUNTER, C. N. \& JONES, O. T. G. (1983). Changes in the cytochrome composition of Rhodopseudomonas sphaeroides grown aerobically, photosynthetically and on dimethyl sulphoxide. Biochemical Joumal 212, 783-790.

Wood, P. M. (1981). Fluorescent gels as a general technique for characterizing bacterial c-type cytochromes. Analytical Biochemistry 111, 235-239.

WU, N. C. \& SchulTz, J. (1975). The prosthetic group of myeloperoxidase. FEBS Letters 60, 141-144.

Yamanake, T., Segnra, M., Takahashi, K. \& ShibaSuKA, M. (1979). Highly purified hydroxylamine oxjdoreductase derived from Nitrosomonas europaea. Joumal of Biochemistry 86, 1101-1108. 